\title{
GW10, a Member of P450 Subfamily Regulates Grain Size and Grain Number in Rice
}

\section{PengLin Zhan ( $D$ 874751264@qq.com )}

South China Agricultural University

\section{Xin Wei}

South China Agricultural University

\section{Zhili Xiao}

South China Agricultural University

\section{Xiaoling Wang}

South China Agricultural University

\section{Shuaipeng $\mathrm{Ma}$}

South China Agricultural University

Shaojun Lin

South China Agricultural University

\section{Fangping Li}

South China Agricultural University

\section{Suhong Bu}

South China Agricultural University

\section{Zupei Liu}

South China Agricultural University

\section{Haitao Zhu}

South China Agricultural University

\section{Guifu Liu}

South China Agricultural University

\section{Guiquan Zhang}

South China Agricultural University

\section{Shaokui Wang}

South China Agricultural University https://orcid.org/0000-0002-7767-4033

\section{Research Article}

Keywords: Rice, GW10, SSSL, Grain size, Grain number, BR

Posted Date: June 7th, 2021 
DOl: https://doi.org/10.21203/rs.3.rs-576630/v1

License: (c) (1) This work is licensed under a Creative Commons Attribution 4.0 International License. Read Full License

Version of Record: A version of this preprint was published at Theoretical and Applied Genetics on August 21st, 2021. See the published version at https://doi.org/10.1007/s00122-021-03939-3. 
$1 \quad$ GW10, a member of P450 Subfamily regulates grain size and grain number in rice

2 Penglin Zhan, Xin Wei, Zhili Xiao, Xiaoling Wang, Shuaipeng Ma, Shaojun Lin, Fangping Li, Suhong

3 Bu, Zupei Liu, Haitao Zhu, Guifu Liu, Guiquan Zhang*, Shaokui Wang*

4 Guangdong Provincial Key Laboratory of Plant Molecular Breeding, State Key Laboratory for

5 Conservation and Utilization of Subtropical Agro-Bioresources, South China Agricultural University,

Guangzhou 510642, China

7

8 * Corresponding authors: Shaokui Wang, Guiquan Zhang

$9 \quad$ E-mail: shaokuiwang@scau.edu.cn,gqzhang@scau.edu.cn

\section{Abstract}

Grain size and grain number play extremely important roles in rice grain yield. Here, we identify GW10, which encodes a P450 subfamily protein and controlls grain size and grain number by using Lemont (tropical japonica) as donor parent and HJX74 (indica) as recipient parent. The GW10 locus was mapped into a $20.1 \mathrm{~kb}$ region on the long arm of Chromosome 10. Lower expression of the $\mathrm{gw} 10$ in panicle is contributed to the shorter and narrower rice grain, and the increased number of grains per panicle. Furthermore, the higher expression levels of some of the brassinosteroid (BR) biosynthesis and response genes are associated with the NIL-GW10, which strongly suggests that the GW10 is a key node in the brassinosteroid-mediated regulation of rice grain shape and grain number.

\section{Key words}

Rice, GW10, SSSL, Grain size, Grain number, BR

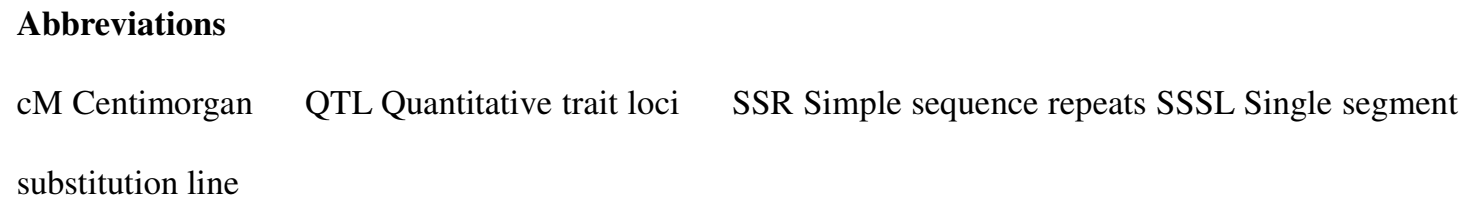

\section{Declarations}

Funding: This work was supported by the Key Projects of Basic Research and Applied Basic Research of Guangdong Province (2019B030302006) and the National Natural Science Foundation of China (32072040).

Conflict of interest: The authors declare that they have no conflict of interest. 
Ethics approval: Not applicable

Consent to participate: Not applicable

Consent for publication: Not applicable

Availability of data and material: Not applicable

Code availability: Not applicable

\section{Author contribution statement}

SW and GZ designed and supervised works. PZ performed most of the experiments, analyzed experimental data and prepared the draft of manuscript. XW, XW, ZX,SM, SL, and FL conducted a part of experiments. SB, ZL, HZ and GL developed the materials. SW wrote the paper. All authors read and approved the final manuscript.

\section{Key message}

A quantitative trait locus GW10 is located on Chromosome 10 by map-based cloning, which encodes a P450 Subfamily protein. The GW10 regulates grain size and grain number in rice involved in the BR pathway.

\section{Introduction}

Rice (Oryza sativa L.) is one of the most crucial cereal foods in the world, which provides over $21 \%$ calorie for the world population and over $76 \%$ calorie for the Asians, and it has been deeply ingrained in their daily lives (Fitzgerald et al. 2009). Grain yield controlling factors include grain weight, grain number per panicle, and panicle number per plant (Zuo et al. 2014). Grain size plays a key role in grain weight, and it is one of the most frequently selected traits during domestication and breeding (Meyer et al. 2013).

Grain weight and grain size are controlled mainly by the grain length, grain width and grain thickness. A number of major quantitative trait loci (QTLs) controlling grain size have been successfully cloned and described. Grain size could be controlled by transcriptional regulators including GW8 (Wang et al. 2012), GLW7 (Si et al. 2016), GS2 (Hu et al. 2015), GL4 (Wu et al. 2017), MADS1 (Yu et al. 2018), GS9 (Zhao et al. 2018) and GW6a (Song et al. 2015; Li et al. 2019). Previously, some major QTLs such as TGW3 (Hu et al. 2018), GS5 (Li et al. 2011), GW5 (Liu et al. 2017) TGW6 (Ishimaru et al., 2013), 
GW6 (Shi et al., 2020) and GS9 (Zhao et al. 2018) were proved to change grain size by phytohormone signals. In the auxin signaling pathway, TGW6 encodes an indole-3-acetic acid (IAA)-glucose hydrolase activity protein and negatively regulates grain size. TGW3 protein interacts with OsARF4 (AUXIN RESPONSE FACTOR 4) repressing downstream auxin response genes' expression (Ishimaru et al. 2013; Hu et al. 2018). GW6 encodes a GA-regulated GAST family protein and positively regulates grain width (Shi et al. 2020). The other three major QTLs of grain size, GS3, GW2 and GL3.1, are involved in Gprotein signaling pathway, ubiquitin-proteasome pathway and cell cycle regulation, respectively (Song et al. 2007; Mao et al. 2010; Qi et al. 2012). In the BR signaling pathway, GS5 binds to OsBAK1-7 affecting BR signaling and grain size (Li et al. 2011). GW5 protein represses GSK2 activity resulting in the altered expression of BR-response genes (Liu et al. 2017). GSK2 influences BR signaling pathway by the phosphorylates OsBZR1 and DLT (Tong et al. 2012). GS9 interacts with OsOFP14 and OsOFP8, whereas OsGSK2 interacts and phosphorylates OsOFP8 affecting the BR signaling pathway (Zhao et al. 2018).

The cytochrome P450 family is one of the biggest protein families in plants. In several sequenced angiosperms, the CYP genes constitute up to $1 \%$ of the total gene annotations of each plant species (Nelson et al. 2004). Several P450 genes have been studied to participate in biosynthesis and catabolism of phytohormones (Mizutani 2010). ELL1 encodes a cytochrome P450 monooxygenase, and influences chlorophyll contents and ROS accumulation (Cui et al. 2021). CYP701A and CYP88A regulates Gibberellins (GAs) biosynthesis to influence seed germination and shoot growth in Arabidopsis (Helliwell et al. 1998; Helliwell et al. 2001). Some P450 genes regulates Brassinosteroids (BRs) biosynthesis to influence plant growth development and regulates Strigolactones (SLs) biosynthesis to influence seed germination of root parasitic plants (Mizutani 2012). In rice, DWARF11 (D11)/ CPB1 IGNS4, encoding cytochrome $\mathrm{P} 450$ proteins are involved in grain size control and brassinosteroid (BR) biosynthesis pathway (Tanabe et al. 2005; Wu et al. 2016; Zhou et al. 2017).

In this study, we identified a P450 subfamily gene, named as GW10 (Grain Width gene on Chromosome 10), which controlled grain size and grain number. The near-isogenic line $g w 10$ (NIL-gw10) produces more grain number per panicle than that of the NIL-GW10 line, while the grain size of the NIL$g w 10$ is smaller than that of the NIL-GW10. The expression levels of the BR biosynthesis and response genes, such as $D 2, D W A R F, C P D, B Z R 1$ and $D L T$, are higher in the NIL- GW10 than those in the NILgw10, which suggests that GW10 might be involved in the BR signaling pathway to regulate grain size 
and grain number in rice. The GW10 might be a potential target for rice breeding by design.

\section{Materials and methods}

\section{Plant materials and growth conditions}

We have developed a library with 2360 SSSLs by using seven AA genome Oryza species as donor parent and Huajingxian74 (HJX74), an elite xian (indica) cultivar from South China as recipient parent. Each of the SSSLs carries only one substitution segment from donor parents on the HJX74 genetic background (Zhang 2019). We generated a SSSL carrying qgw10 Lemont (NIL-gw10) which was derived from the Lemont $\times$ HJX74 $F_{1}$ hybrid with an additional five times backcrossing to HJX74 in the SSSL library. The NIL-gw10 carried an substitution segment from Lemont. The segment was determined between marker RM258-RM147 on chromosome 10. All of the plant materials were planted in paddy field in South China Agriculture University, Guangzhou, China $\left(23^{\circ} 07^{\prime} \mathrm{N}, 113^{\circ} 15^{\prime}\right.$ E). Seedings were transplanted in paddy field after about 25 days, and each line was grown in a single row of ten plants (Fang et al., 2019).

\section{Genetic analysis and fine mapping of the GW10}

We developed $\mathrm{F}_{2}$ population from the cross between NIL-GW10 (HJX74) and NIL-gw10. We selected polymorphic simple sequence repeat (SSR) markers between these two NILs according to the rice linkage map (http://www.gramene.org). A series of Indel and SNP polymorphic markers which were designed according to the sequence variations between the japonica cultivar Nipponbare (https://rapdb.dna.affrc.go.jp) and HJX74 (http://192.168.87.153/) were used for GW10 fine mapping (Li et al., 2021) (Table S1). The linkage analysis between the markers and the grain width locus for the $\mathrm{F}_{2}$ population mapped the $G W 10$ gene to a chromosome 10 region flanked by polymorphic markers.

\section{Genomic DNA extraction and PCR analysis}

The fresh young leaves of individual rice were collected into $2 \mathrm{ml}$ centrifuge tube and then were grounded in liquid nitrogen. The solution used in the experiment has been reported previously (Chen et al., 2015). The PCR program for the initial denaturing step was at $94^{\circ} \mathrm{C}$ for $5 \mathrm{~min}$, followed by 38 cycles for $30 \mathrm{~s}$ at $94{ }^{\circ} \mathrm{C}, 30 \mathrm{~s}$ at $55^{\circ} \mathrm{C}, 45 \mathrm{~s}$ at $72{ }^{\circ} \mathrm{C}$, with a final extension at $72^{\circ} \mathrm{C}$ for 5 min. The $6 \%$ nondenaturing polyacrylamide gel was used for separating PCR products and the genomic DNA polumorphic analysis was carried out by the silver staining method.

\section{RNA extraction and quantitative real-time PCR}

Total RNA was isolated from young panicles of NIL-GW10 and NIL- $g w 10$ by using TRIZOL 
reagent (Invitrogen) following the manufacturer's instruction. First-strand cDNA was reverse transcribed from $5 \times$ All-In-One RT MasterMix (with AccuRT Genimic DNA Removal Kit), which was implemented for detected gene semi-quantitative/quantitative analysis with specific primers. Gene transcript levels were measured by qRT-PCR using the ABI 7500 real-time PCR system while actin gene was used as internal control. Each qRT-PCR program was performed in total volume of $20 \mu \mathrm{l}$ schema containing $1 \times$ SYBR Green Master Mix. Each experiment was repeated three times, and the relative quantitative method was used to evaluate quantitative variation. The qRT-PCR reaction program was directed at $94^{\circ} \mathrm{C}$ for $10 \mathrm{~min}$, followed by 40 cycles at $94^{\circ} \mathrm{C}$ for $30 \mathrm{~s}, 60^{\circ} \mathrm{C}$ for $1 \mathrm{~min}$. Primers for experiment were referred to the Table S2.

\section{Gene cloning and sequence analysis}

Candidate genes were cloned by the gene specific primers, and the primers were produced by using HJX74 genome sequence as reference. The KOD FX DNA polymerase (TOYOBO, Japan) was employed in the PCR assay. The information of ORFs (Open Reading Frames) and its corresponding predicted protein sequence was obtained from NCBI (https://www.ncbi.nlm.nih.gov/). The multiple DNA and protein sequences of candidate genes in NIL-GW10 and NIL-gw10 were aligned by software DNAman. The phylogenetic analysis was presented by MAGA6 based on the Neighbor-Joining (NJ) model and the bootstrap values were estimated with 1000 replicates.

\section{Vector construction and rice transformation}

To generate the Cas9 vector for GW10, two targets were designed in the ORF of candidate gene. The intermediate SK-gRNA vector was constructed using the isocaudamer ligation method. The SKgRNA-target 1 and SK-gRNA-target2 were digested with KpnI/NheI and XbaI/BglII, respectively. The digested products were gel purified and ligated into pC1300-Cas9 binary vector (digested with KpnI/BamHI). The vector was transformed into Agrobacterium tumefaciens EHA105 cells, and resulting strains were implemented to transform the callus of HJX74 (Hu et al., 2018).

\section{Agronomic traits evaluation and statistical analysis}

Agronomic traits were evaluated in the different period of plant growth. Plant height and number of effective tillers were measured at the maturity stage. Heading date was calculated at the time when the first panicle sprouting. Meanwhile, panicle length, primary branch, secondary branch, 1000-grain weight, grain length and grain weight were investigated after the rice harvest at the maturity stage. Grain length and grain weight were measured by Microtek ScanWizard EZ scanner V-2.140 and Wan Shen grain 
analyzer software. Each set of groups was recorded about ten plants. All the data was analyzed using IBM SPASS statistic 20. The significance was accepted at $P<0.05$ and $P<0.01$.

\section{Results}

\section{Comparison of grain and panicle traits of NIL-GW10 and NIL-gw10}

Grain size and grain number per panicle play extremely important roles in rice grain yield (Li et al. 2019). We constructed a set of SSSLs by crossing Lemont (donor parent ) and HJX74 (recipient parent). Each of the SSSLs carries only one substitution segment from the Lemont on the genetics background of HJX74. Then QTL analysis showed that the presene of a minor grain size locus $q G W 10$ on the long arm of chromosome 10. The near-isogenic line gw10 which was drived from Lemont produced significantly shorter grain length than that of NIL-GWIO (namely HJX74) (Fig. 1c and d) and the plant of NIL-gw10 is slightly higher than that of the NIL-GW10 (Fig. 1a). The grain width of NIL-gw10 was also markedly narrower than that of NIL-GW10 and the 1000-grain weight decreased accordingly in NIL-gw10 (Fig. 1c,e and f ). In addition, plant height (Fig. 2b) and panicle length (Fig. 2d) of NIL-gw10 were markedly higher and longer than that of NIL-GW10. There were no difference between the two NILs in the heading date (Fig. 2a) and number of tillers (Fig. 2c). Moreover, the secondary branch per panicle of NIL-gw10 were much more than that of NIL-GW10, which resulted in an increased number of grains per plant (Fig. 2e and f). These results indicated that the introgressed substitution segment from Lemont in HJX74 contributed to the decrease in grain length and grian width, meanwhile, to the increase in the grain number per panicle.

\section{Genetic analysis and mapping of $G W 10$}

To study the genetic factor for $q G W 10$, we crossed NIL-GW10 with NIL-gw10 to generate $\mathrm{F}_{2}$ population. The grain width was used as a target trait. The genotype and phenotype of $F_{2}$ population conformed to a segregation ratio $1: 2: 1\left(\chi^{2}=0.11<\chi^{2} 0.01,2=9.21\right)$. The inheritance patterns of the $F_{2}$ plants indicated that a semidominant $q G W 10$ allele from HJX74 controlled grain size (Fig. 1b).

A $3200 \mathrm{~F}_{2}$ segregants was bred from the cross between the NIL-gw10 and HJX74. A subsequent high resolution map was constructed on the basis of the $\mathrm{F}_{2}$ population. The region of $q G W 10$ was narrowed down to a $20.1 \mathrm{~kb}$ flanked by marker Z4 and Z5 on chromosome 10 (Fig.3a and b). The segment contains only 1 predicted open reading frames $(O R F 1)$ and is very close to the start codon of $O R F 2$ according to the HJX74 genome(Fig. 3c). The ORF1 and ORF2 correspond to Os $10 g 0515400$ and Os $10 g 0515900$ in Nipponbare respectively. The expression profiles of $O R F 1$ in various tissues of NIL- 
GW10 and NIL-gw10 were tested by RT-PCR analysis. No expression of ORF2 was detected at all the tissues (Fig. S1b), while the qPCR analysis indicated that $O R F 1$ was expressed in root, stem, leaf and developing panicles (Fig. S1a). The expression was significantly reduced in the young panicle of the NIL-gw10 compared to that of the NIL-GW10 especially in the panicles of $6 \mathrm{~cm}$ in length (Fig. 3d). It is strongly suggested $O R F 1$ is the candidate gene for GW10. The ORF1(Os10g0515400) encodes a P450 subfamily protein CYP89A2 (Fig. S2). The sequence comparison of GW10 in Lemont and HJX74 revealed four polymorphisms in the promoter region and two polymorphisms in the coding sequence. There is one synonymous polymorphism (C $792 \mathrm{G}$ ) and an in frame 3 bp Indel in the coding sequence. A $3326 \mathrm{bp}$ Indel in the uptream of the coding region of Os10g0515400 gene locus was probablely associated with the down regulation of the gene in NIL-gw10 (Fig. 3c and Table S3 ). The role of Os $10 g 0515400$ in rice development is not clear. Phylogenetic analysis of GW10 protein indicates that it is ubiquitous in the poaceae (Fig. S3), suggests that GW10 might play a vital role in the poaceae development.

\section{GW10 controls grain size and grain number}

We generated the GW10 knockout transgenic plants by the CRISP/cas9 genome editing system in HJX74 and obtained homozygous transgenic $\mathrm{T}_{2}$ plants (Fig. S4 and S5). The grain length and grain width were examined in the NIL-GW10, NIL-gw10 and KO-GW10 lines at the mature stage. The NIL-GW10 line produced grains with length of $8.40 \mathrm{~mm}$ and width of $2.82 \mathrm{~mm}$, while the NIL-gw 10 line produced grains with length of $7.93 \mathrm{~mm}$ and width of $2.72 \mathrm{~mm}$. The KO-GW1O line had a grain length of $7.71 \mathrm{~mm}$ and a grain width of $2.71 \mathrm{~mm}$ (Fig.4a, c and d). These results indicate that the grain size of KO-GW1O line was significantly smaller than that of NIL-GW10 and NIL-gw10. The 1000-grain weight of KOGW10 line decreased 5.5\% and 14.6\% compared to that of NIL-gw10 and NIL-GW10, respectively (Fig. 4e). Interestingly, the number of grains per plant was obviously different in the three lines. The KOGW10 line had much more grain number compared to the NIL-GW10 and NIL-gw10 line (Fig.4b and f). The heading date, the number of tillers and the panicle length of KO-GW10 were no difference compared to those of NIL-GW10 (Fig. S5a, b, d and e). The plant height of KO-GW10 was higher than that of NILGW10 with $P=0.03$ (Fig. S5c). These results indicate that the GW10 protein regulates grain size and grain numbers in rice.

\section{GW10 involved in the BR biosynthesis pathway}

The GW10 encodes a cytochrome P450 subfamily 89A2 homology protein. In the previous reports, 
a new allele of DWARF2 (D2), smg11, encoding a cytochrome P450 protein which controls grain size and grain number by involving in brassinosteroid (BR) biosynthesis pathway (Fang et al. 2016). Our meterials have similar phenotypes to those of the $\operatorname{smg} 11$. Therefore, the $G W 10$ might participate in the BR biosynthesis pathway. We examined the expression levels of BR biosynthetic and BR-signaling genes in the $0.2 \mathrm{~cm}$ young panicle. The expression levels of $D 2, D W A R F, C P D, B Z R 1$ and DLT of NIL-GW10 were markedly higher than those of NIL-gw10, while the expression levels of $B U 1$ and GSK2 were significantly lower in NIL-GW10 than those in NIL-gw10. The BIRl expression level was not altered in the NIL-gw10 and NIL-GW10 lines (Fig. 5a). The expression levels of GS2 and GS9 were no significant difference in the two NILs, but the GW5 and DEP2 expression were significantly different with $P<0.01$ and $P<0.05$ levels, respectively (Fig. 5b). These results indicated that the GW10 protein might regulate BR-signaling genes by influencing $G W 5$ expression.

\section{Discussion}

It was difficult to determine the precise location of QTLs in rice genome by using the $\mathrm{F}_{2}$ plants, Recombinant Inbred Lines (RILs), Backcross Inbred Lines (BILs) and Doubled Haploid Lines (DHLs) (Ashikari et al. 2006). Consequently, the development of Nearly Isogenic Lines (NILs), Chromosome Fragment Substitution Line (CSSL) and Single Segment Substitution Line (SSSL) were necessary for QTL fine mapping and gene cloning (Ashikari et al. 2006; Guo et al. 2016; Zhou et al.2017; Wang et al. 2018; Yang et al. 2018; Luan et al. 2019; Tan et al. 2020; Tan et al. 2021), especially for the minor genes. The additive effect value of was GW10 lower than some major genes for grain size, such as gs3, GW5 and GW7. The SSSLs were excellent material for the cloning and functional analysis of the GW10. Many QTLs for grain size have been identified and distributed across nearly all the 12 chromosomes in rice. However, only a few QTLs for grain size were reported on chromosome 10 (Li et al. 2019). The GW10 was located on the long arm of chromosome 10 and was incomplete dominant inheritance (Fig. 1b). The qPCR was performed to examine the expression level of GW10 in various rice tissues, including roots, stems, leave and panicles in different growth stages. The GW10 is constitutively transcribed in all tested tissues (Fig 3d and Fig S1a) The grain size, plant height, number of secondary branch and grain number per panicle were difference between the line of NIL-GW10 and NIL-gw10 (Fig. 1 and 2), which illustrated that the $G W 10$ was a pleiotropic gene in rice.

The GW10 encodes a P450 Subfamily 89A2 homology protein. The cytochrome P450 family is one of the biggest protein families in plants and several P450 proteins were found that invoved in the 
controlling of rice grain size. The $C P B 1$ which encoding a cytochrome $\mathrm{P} 450$ superfamily protein effected grain size and other agronomic traits in rice. The OE-CPB1 transgenic plants showed increasing grain length while the RNAi-CPB1 transgenic plants showed smaller grain size and semidwarf in stature $(\mathrm{Wu}$ et al. 2016; Shi et al. 2018). A small grain (smg11) mutant in rice exhibited more secondary panicle branches, small grains and increased number of grains per panicle (Fang et al. 2016). The NBG4, also reported as Small grain 4 (Shi et al. 2015)/ Dwarf 11 (Tanabe et al. 2005)/ Clustered spokelets4 (Guo et al. 2014)/ GNS4 (Zhou et al. 2017) encodes a cytochrome P450 (CYP724B1). A 10-bp deletion in the CDS resulted in the lower expression of $n b g 4$ than that of the NBG4 in ZH11 and the deletion was contributed to the grain shape (Tong et al. 2018). In this study, the lower expression level of $g w 10$ in young panicle was associated with the smaller grain size and the more grain number in NIL-gw10 (Fig. 1 and Fig. 2f). Additionally, the grain size of the KO-GW10 line was smaller than that of the NIL-gw10 and of the NIL-GW10 (Fig. 4a) while the grain number of the KO-GW10 line was obviously increased comparing with NIL-gw10 and NIL-GW10 (Fig. 4b). It implied that the four variations in the promoter (Fig. 3c, Table S3) would be the key potential cis-regulators for the smaller grain size in NIL-gw10.

It was reported that the CYP724B1 could control grain size by affectting BR-related genes (Shi et al. 2015; Zhou et al. 2017). BRs regulate various aspects of plant development, including root development, anther and pollen development, stem elongation and cellulose biosynthesis in plants (Yang et al. 2011). Our study demonstrated that GW10 is a key factor in the BRs mediated regulation of rice grain size (Fig 5a). GSK2 is one of the orthologs of BIN2 which plays crucial roles in the BR signaling pathway, and GSK2 could inhibit the activities of $O S B Z R 1$ and $D L T$ by phosphorylating the two proteins (Tong et al. 2012). BU1 protein acts as a positive regulator of the BR pathway and participates in BR signaling pathways through $O s B R I 1$ and RGAl (Tanaka et al. 2009). GW5 could physically interact with GSK2 and inhibit its kinase activities toward BZRI and DLT (Liu et al. 2017). In NIL-gw10, the expression of GW5 was significantly higher than that of NIL-GW10. Beside, a series of GSK2 downstream genes including of $B Z R 1, D L T, D 2$, wwarf and $C P D$ expression was significantly down regulated (Fig. 5a), which implies GW10 induces the decline of GSK2 expression by influencing GW5. DEP2 mainly affects the rapid elongation of rachis and primary and secondary branches (Li et al. 2010) . The expression level of DEP2 is higher in NIL-gw10 than in NIL-GW10, which suggests that GW10 should involve in the regulatory of $D E P 2$ for grain size and secondary branch (Fig. 5b). The rice grain size is regulated by a complicated network. Although recent studies have identified some key grain size 
genes and molecular pathways, the complete regulatory network for grain size is poorly understood (Li et al. 2019). Our work provides a piece of this complex puzzle and make a contribution to the enrichment of breeding germplasm resources bases.

\section{References}

Ashikari M, Matsuoka, M. (2006). Identification, isolation and pyramiding of quantitative trait loci for rice breeding. Trends Plant Sci, 11(7):344-350

Babineau M, Mahmood K, Mathiassen SK, Kudsk P, Kristensen M (2017) De novo transcriptome assembly analysis of weed Apera spica-venti from seven tissues and growth stages. Bmc Genomics 18:128

Chen J, Li X, Cheng C, Wang Y, Qin M, Zhu H, Zeng R, Fu X, Liu Z, Zhang G (2015) Characterization of epistatic interaction of QTLs $L H 8$ and $E H 3$ controlling heading date in rice. Sci Rep 4:4263

Cui Y, Peng Y, Zhang Q, Xia S, Ruan B, Xu Q, Yu X, Zhou T, Liu H, Zeng D, Zhang G, Gao Z, Hu J, Zhu L, Shen L, Guo L, Qian Q, Ren D (2021) Disruption of EARLY LESION LEAF 1, encoding a cytochrome P450 monooxygenase, induces ROS accumulation and cell death in rice. Plant J 105:942-956

Fang N, Xu R, Huang L, Zhang B, Duan P, Li N, Luo Y, Li Y (2016) SMALL GRAIN 11 controls grain size, grain number and grain yield in rice. Rice 9:64

Fitzgerald M A, McCouch S R, Hall R D (2009) Not just a grain of rice: The quest for quality. Trends Plant Sci 14:133-139

Guo J, Xu X, Li W, Zhu W, Zhu H, Liu Z, Luan X, Dai Z, Liu G, Zhang Z, Zeng R, Tang G, Fu X, Wang S, Zhang G (2016) Overcoming inter-subspecific hybrid sterility in rice by developing indicacompatible japonica lines. Sci Rep 6:26878

Guo M, Yang Y, Liu M, Meng Q, Zeng X, Dong L, Tang S, Gu M, Yan C (2014) Clustered spikelets 4, encoding a putative cytochrome $\mathrm{P} 450$ protein CYP724B1, is essential for rice panicle development. Chin Sci Bull 59:4050-4059

Helliwell CA, Sheldon CC, Olive MR, Walker AR, Zeevaart JA, Peacock WJ, Dennis ES (1998) Cloning of the Arabidopsis ent-kaurene oxidase gene GA3. Proc. Natl Acad Sci 95:9019-9024

Helliwell CA, Chandler PM, Poole A, Dennis ES, Peacock WJ (2001) The CYP88A cytochrome P450, ent-kaurenoic acid oxidase, catalyzes three steps of the gibberellin biosynthesis pathway. Proc Natl 
301

302

303

Hu J, Wang Y, Fang Y, Zeng L, Xu J, Yu H, Shi Z, Pan J, Zhang D, Kang S, Zhu L, Dong G, Guo L, Zeng D, Zhang G, Xie L, Xiong G, Li J, Qian Q (2015) A rare allele of GS2 enhances grain size and grain yield in rice. Mol Plant 8:1455-1465

Hu X, Meng X, Liu Q, Li J, Wang K (2018) Increasing the efficiency of CRISPR-Cas9-VQR precise genome editing in rice. Plant Biotechnol J 16:292-297

Hu Z, Lu S, Wang M, Wang H, He H, Sun J, Sun L, Liu J, Liu X, Jiang L, Xin X, Kong W, Chu C, Xue H, Yang J, Luo X (2018) A novel QTL $q T G W 3$ encodes the GSK3/SHAGGY-Like kinase OsGSK5/OsSK41 that interacts with OsARF4 to negatively regulate grain size and weight in rice. Mol Plant 11:736-749

Ishimaru K, Hirotsu N, Madoka Y, Murakami N, Hara N, Onodera H, Kashiwagi T, Ujiie K, Shimizu B, Onishi A, Miyagawa H, Katoh E (2013) Loss of function of the IAA-glucose hydrolase gene TGW6 enhances rice grain weight and increases yield. Nat Genet 45:707-711

Li F, Gao Y, Wu B, Cai Q, Zhan P, Yang W, Shi W, Li X, Yang Z, Tan quan, Luan X, Zhang G, Wang S (2021) High-Quality de novo genome assembly of Huajingxian74, a recipient parent of single segment substitution lines. Rice Sci 28:109-113

Li F, Liu W, Tang J, Chen J, Tong H, Hu B, Li C, Fang J, Chen M, Chu C (2010) Rice DENSE AND ERECT PANICLE 2 is essential for determining panicle outgrowth and elongation. Cell Res 20:838-849

Li N, Xu R, Li Y (2019) Molecular networks of seed size control in plants. Annu Rev Plant Biol 70:435463

Li Y, Fan C, Xing Y, Jiang Y, Luo L, Sun L. (2011) Natural variation in GS5 plays an important role in regulating grain size and yield in rice. Nat Genet 43:1266-1269

Liu J, Chen J, Zheng X, Wu F, Lin Q, Heng Y, Tian P, Cheng Z, Yu X, Zhou K, Zhang X, Guo X, Wang J, Wang H, Wan J (2017) GW5 acts in the brassinosteroid signalling pathway to regulate grain width and weight in rice. Nat Plants 3:17043

Luan X, Dai Z, Yang W, Tan Q, Lu Q, Guo J, Zhu H, Liu G, Wang S, Zhang G (2019) Breeding by design of CMS lines on the platform of SSSL library in rice. Mol Breed 39:126

Mao H, Sun S, Yao J, Wang C, Yu S, Xu C, Li X, Zhang Q (2010) Linking differential domain functions of the GS3 protein to natural variation of grain size in rice. Proc Natl Acad Sci 107:19579-19584 
Meyer RS, Purugganan MD (2013) Evolution of crop species: Genetics of domestication and diversification. Nat Rev Genet 14:840-852

Mizutani M, Ohta D (2010) Diversification of P450 genes during land plant evolution. Annu. Rev. Plant Biol. 61: 291-315

Mizutani M (2012) Impacts of diversification of cytochrome P450 on plant metabolism. Biol Pharm Bull $35: 824-832$

Nelson DR, Schuler MA, Paquette SM, Reichhart DW, Bak S (2004). Comparative genomics of rice and arabidopsis. analysis of 727 cytochrome p450 genes and pseudogenes from a monocot and a dicot. Plant Physiol 135:756-772

Qi P, Lin YS, Song XJ, Shen JB, Huang W, Shan JX, Zhu MZ, Jiang L, Gao JP, Lin HX (2012) The novel quantitative trait locus GL3.1 controls rice grain size and yield by regulating Cyclin-T1;3. Cell Res 22:1666-1680

Shi CL, Dong NQ, Guo T, Ye WW, Shan JX, Lin HX (2020) A quantitative trait locus GW6 controls rice grain size and yield through the gibberellin pathway. Plant J 103:1174-1188

Shi Z, Rao Y, Xu J, Hu S, Fang Y, Yu H, Pan J, Liu R, Ren D, Wang X (2015) Characterization and cloning of SMALL GRAIN 4, a novel DWARF11 allele that affects brassinosteroid biosynthesis in rice. Sci Bull 60:905-915.

Si L, Chen J, Huang X, Gong H, Luo J, Hou Q, Zhou T, Lu T, Zhu J, Shangguan Y, Chen E, Gong C, Zhao Q, Jing Y, Zhao Y, Li Y, Cui L, Fan D, Lu Y, Weng Q, Wang Y, Zhan Q, Liu K, Wei X, An K, An G, Han B (2016) OsSPL13 controls grain size in cultivated rice. Nat Genet 48:447-456

Song X, Huang W, Shi M, Zhu M, Lin H (2007) A QTL for rice grain width and weight encodes a previously unknown RING-type E3 ubiquitin ligase. Nat Genet 39:623-630

Song XJ, Kuroha T, Ayano M, Furuta T, Nagai K, Komeda N, Segami S, Miura K, Ogawa D, Kamura T, Suzuki T, Higashiyama T, Yamasaki M, Mori H, Inukai Y, Wu J, Kitano H, Sakakibara H, Jacobsen SE, Ashikari M (2015) Rare allele of a previously unidentified histone H4 acetyltransferase enhances grain weight, yield, and plant biomass in rice. Proc Natl Acad Sci $112: 76-81$

Tan Q, Zou T, Zheng M, Ni Y, Luan X, Li X, Yang W, Yang Z, Zhu H, Zeng R, Liu G, Wang S, Fu X, Zhang G (2020) Substitution mapping of the major quantitative trait loci controlling stigma exsertion rate from Oryza glumaepatula. Rice 13:37 
Tan Q, Wang C, Luan X, Zheng L, Ni Y, Yang W, Yang Z, Zhu H, Zeng R, Liu G, Wang S, Zhang G. 2021. Dissection of closely linked QTLs controlling stigma exsertion rate in rice by substitution mapping. Theor Appl Genet 134: 1253-1262

Tanabe S, Ashikari M, Fujioka S, Takatsuto S, Yoshida S, Yano M, Yoshimura A, Kitano H, Matsuoka M, Fujisawa Y, Kato H, Iwasaki Y (2005) A novel cytochrome p450 is implicated in brassinosteroid biosynthesis via the characterization of a rice dwarf mutant, dwarf11, with reduced seed length. Plant Cell 17:776-790

Tanaka A, Nakagawa H, Tomita C, Shimatani Z, Ohtake M, Nomura T, Jiang C, Dubouzet JG, Kikuchi S, Sekimoto H, Yokota T, Asami T, Kamakura T, Mori M (2009) BRASSINOSTEROID UPREGULATED1, encoding a Helix-Loop-Helix protein, is a novel gene involved in brassinosteroid signaling and controls bending of the lamina joint in rice. Plant Physiol 151:669680

Tong H, Liu L, Jin Y, Du L, Yin Y, Qian Q, Zhu L, Chu C (2012) DWARF and LOW-TILLERING acts as a direct downstream target of a GSK3/SHAGGY-Like kinase to mediate brassinosteroid responses in rice. Plant Cell 24:2562-2577

Tong X, Wang Y, Sun A, Bello B, Ni S, Zhang J (2018). Notched belly grain 4, a novel allele of $d$ warf 11, regulates grain shape and seed germination in rice (Oryza sativa L.). Int J of Mol Sci 19:4069

Wang S, Li S, Liu Q, Wu K, Zhang J, Wang S, Wang Y, Chen X, Zhang Y, Gao C, Wang F, Huang H, Fu X (2015) The OsSPL16-GW7 regulatory module determines grain shape and simultaneously improves rice yield and grain quality. Nat Genet 47:949-954

Wang S, Wu K, Yuan Q, Liu X, Liu Z, Lin X, Zeng R, Zhu H, Dong G, Qian Q, Zhang G, Fu X (2012) Control of grain size, shape and quality by $O s S P L 16$ in rice. Nat Genet 44:950-954

Wang X, Jin L, Zhu H, Wang S, Zhang G, Liu G (2018) QTL epistatic analysis for yield components with single-segment substitution lines in rice. Plant Breed 137:346-354

Wu W, Liu X, Wang M, Meyer RS, Luo X, Ndjiondjop M, Tan L, Zhang J, Wu J, Cai H, Sun C, Wang X, Wing RA, Zhu Z (2017) A single-nucleotide polymorphism causes smaller grain size and loss of seed shattering during African rice domestication. Nat Plants 3:17064

Wu Y, Fu Y, Zhao S, Gu P, Zhu Z, Sun C, Tan L (2016) CLUSTERED PRIMARY BRANCH 1, a new allele of DWARF11, controls panicle architecture and seed size in rice. Plant Biotechnol J 14:377386 
Yang C, Zhang C, Lu Y, Jin J, Wang X (2011) The Mechanisms of Brassinosteroids' Action: From Signal Transduction to Plant Development. Mol Plant 4:588-600

Yang Z, Jin L, Zhu H, Wang S, Zhang G, Liu G (2018) Analysis of Epistasis among QTLs on Heading Date based on Single Segment Substitution Lines in Rice. Sci Rep 8:3059

Yu J, Miao J, Zhang Z, Xiong H, Zhu X, Sun X, Pan Y, Liang Y, Zhang Q, Abdul Rehman RM, Li J, Zhang H, Li Z (2018) Alternative splicing of $O s L G 3 b$ controls grain length and yield in japonica rice. Plant Biotechnol J 16:1667-1678

Zhang GQ (2019) The platform of breeding by design based on the SSSL library in rice. Hereditas (Beijing) 41:754-760 (In Chinese with English abstract)

Zhao D, Li Q, Zhang C, Zhang C, Yang Q, Pan L, Ren X, Lu J, Gu M, Liu Q (2018) GS9 acts as a transcriptional activator to regulate rice grain shape and appearance quality. Nat Commun 9:1240

Zhou Y, Tao Y, Zhu J, Miao J, Liu J, Liu Y, Yi C, Yang Z, Gong Z, Liang G (2017) GNS4, a novel allele of DWARF11, regulates grain number and grain size in a high-yield rice cultivar. Rice 10:34

Zhou Y, Xie Y, Cai J, Liu C, Zhu H, Jiang R, Zhong Y, Zhang G, Tan B, Liu G, Fu X, Liu Z, Wang S, Zhang G, Zeng R (2017b) Substitution mapping of QTLs controlling seed dormancy using single segment substitution lines derived from multiple cultivated rice donors in seven cropping seasons. Theor Appl Genet 130:1191-1205

Zhu Y, Zhang Z, Chen J, Fan Y, Mou T, Tang S, Zhuang J (2019) Fine mapping of qTGW10-20.8, a QTL having important contribution to grain weight variation in rice. Crop J 7:587-597

Zuo J, Li J (2014) Molecular genetic dissection of quantitative trait loci regulating rice grain size. Annu Rev Genet 48:99-118

\section{Figure legends}

Fig. 1 Morphological characteristics of NIL-GW10 and NIL-gw10. a. The morphology of the NIL-GW10 and NIL-gw10 plants. Scale bar, $15 \mathrm{~cm}$. b. Frequency distribution of grain width in $\mathrm{F}_{2}$ population. c. Grain shape of NIL-GW10 and NIL-gw10. Scale bar, $5 \mathrm{~mm}$. d. Grain length $(\mathrm{n} \geq 100)$. e. Grain width (n $\geq 100$ ). f. 1000 -grain weight, 500 grains was weighted by electronic balance and the data was converted into 1000-grain weight, 3 repeats. Data in d-f are given as means \pm SD. Student's $t$-test were employed for the evaluation of $P$ value. $* P \leq 0.05,{ }^{* *} P \leq 0.01$. 
421 Fig. 2 The agronomic traits of NIL-GW10 and NIL-gw10. a. Heading date. b. Plant height. c. Number 422 of effective tiller. d. Panicle length. e. Secondary branches per panicle. f. Number of grains per plant. 423 2019ES, the early season in 2019; 2019LS, the late season in 2019 . Values are means \pm SD. $* P \leq 0.05$ $424 * * * P \leq 0.01$

425

Fig. 3 Fine-mapping of GWIO. a. The substitution segment on chromosome 10. b. $q$ GWIO was mapped to $20.1 \mathrm{~kb}$ region between marker $\mathrm{Z} 4$ and $\mathrm{Z} 5$ using $3,200 \mathrm{BC}_{5} \mathrm{~F}_{3}$ plants. The number above the line indicate the number of recombinants between each of the two adjacent markers. Black filled and open bars represent substitution segments homozygous for the Lemont and HJX74 alleles, respectively. Grain width is shown for recombinant plants (I-IV) and NILs. Data are shown as means \pm SD ( $\geq 100)$. c. Allelic variation in the region of candicate gene ORF1 (Os10g0515400) between Lemont and HJX74. d. Expression of $O R F 1$ in NIL-GW10 and NIL- $g w 10.0 .2 \mathrm{~cm}-8 \mathrm{~cm}$, young panicles and the number showed the length of the panicle $(\mathrm{cm})$.

Fig. 4 The effects of $G W 10$ on grain size and grain number. a. The grains from NIL-GW10, NIL-gw10 and target-gene edited NIL-GW10 (KO-GW10). Scale bar, $5 \mathrm{~mm}$. b The grains from NIL-GW10, NILgw10 and KO-GW10. Scale bar, $5 \mathrm{~cm}$. c. Grain length $(\mathrm{n} \geq 100)$. d. Grain width $(\mathrm{n} \geq 100)$. e. 1000-grain weight, 3 repeats. f. number of per plants $(\mathrm{n} \geq 20)$. Values shown in $\mathbf{c}-\mathbf{f}$ are means \pm SD. a, b, c $P \leq 0.05$.

Fig. 5 Transcript levels of BR responsive and BR-related grain size genes in young panicle. a BR responsive genes. b. BR-related grain size genes. Expression is shown relative to that of NIL-GW10 plants, which was set to 1 . Data are shown as means \pm SE. $P$ values from the $t$-test were indicated, $* P \leq$ $0.05, * * \leq 0.01$ 


\section{Figures}

a

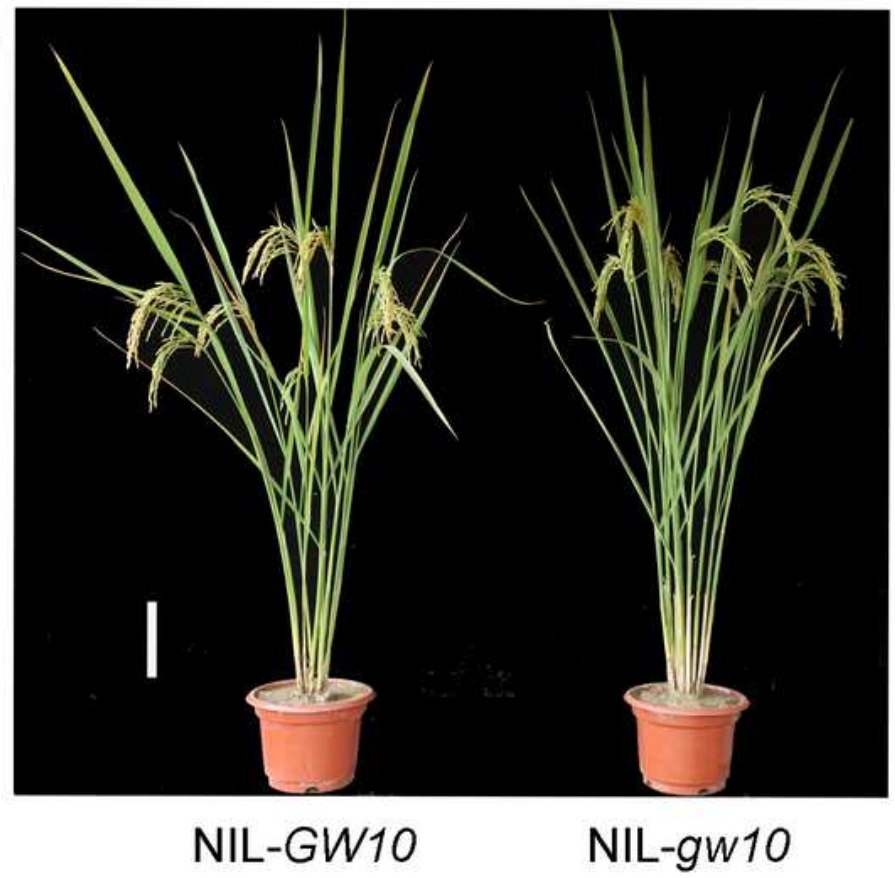

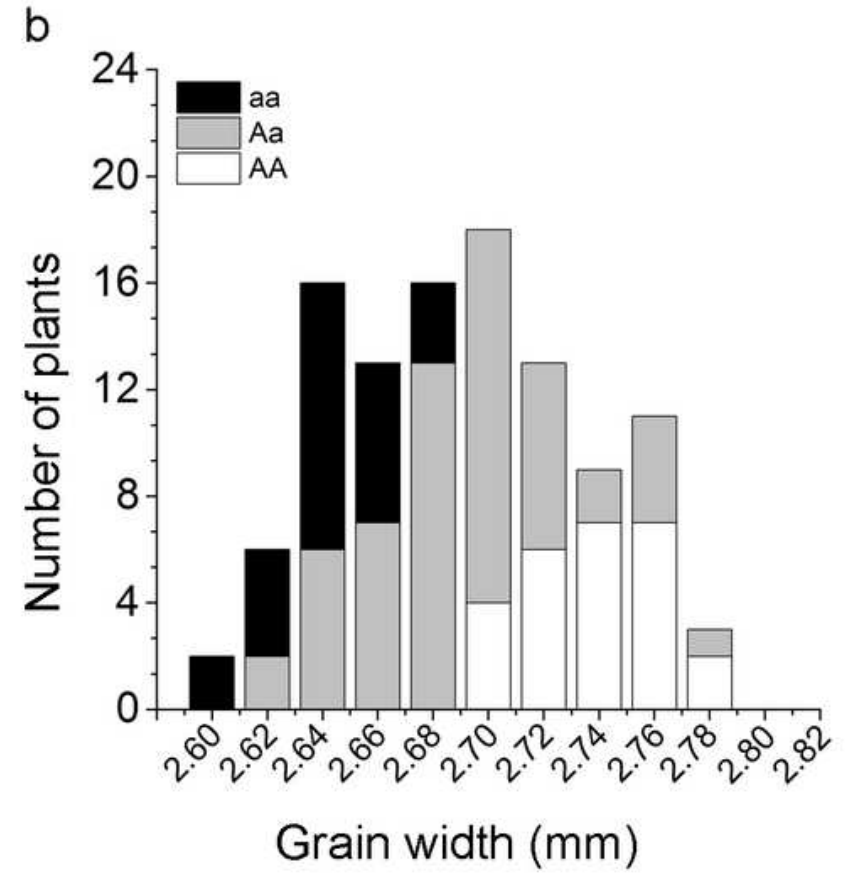

C
NIL-GW10
NIL-gw10

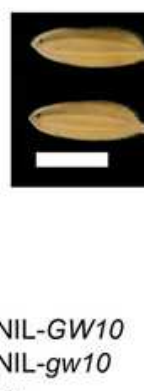

d

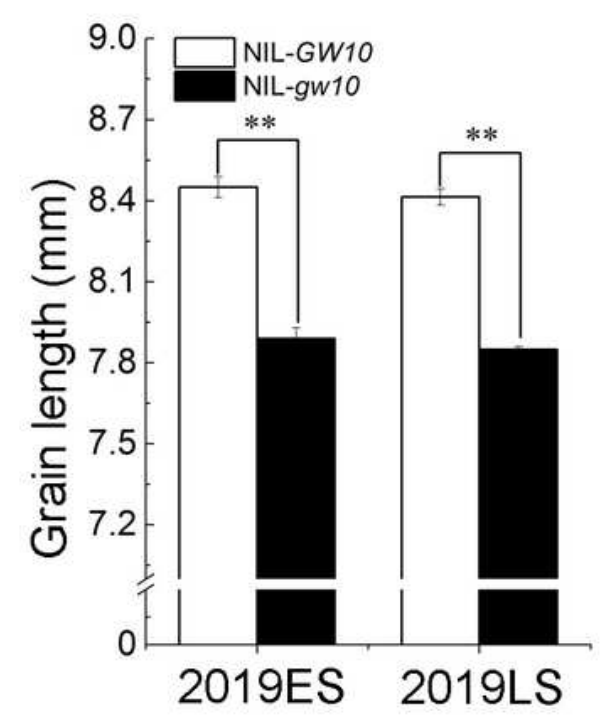

e
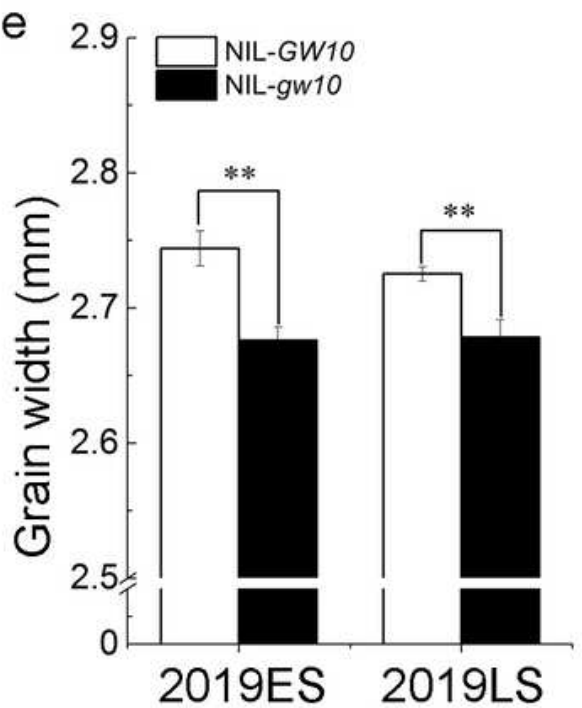
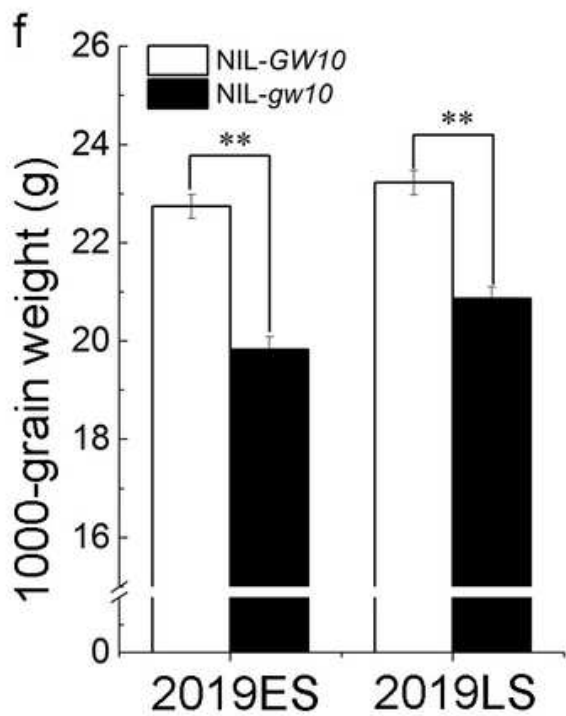

\section{Figure 1}

Morphological characteristics of NIL-GW10 and NIL-gw10. a. The morphology of the NIL-GW10 and NILgw10 plants. Scale bar, $15 \mathrm{~cm}$. b. Frequency distribution of grain width in F2 population. c. Grain shape of NIL-GW10 and NIL-gw10. Scale bar, 5 mm. d. Grain length $(n \geq 100)$. e. Grain width $(n \geq 100)$. f. $1000-$ 
grain weight, 500 grains was weighted by electronic balance and the data was converted into 1000-grain weight, 3 repeats. Data in d-f are given as means \pm SD. Student's t-test were employed for the evaluation of $P$ value. ${ }^{*} \mathrm{P} \leq 0.05, * * P \leq 0.01$
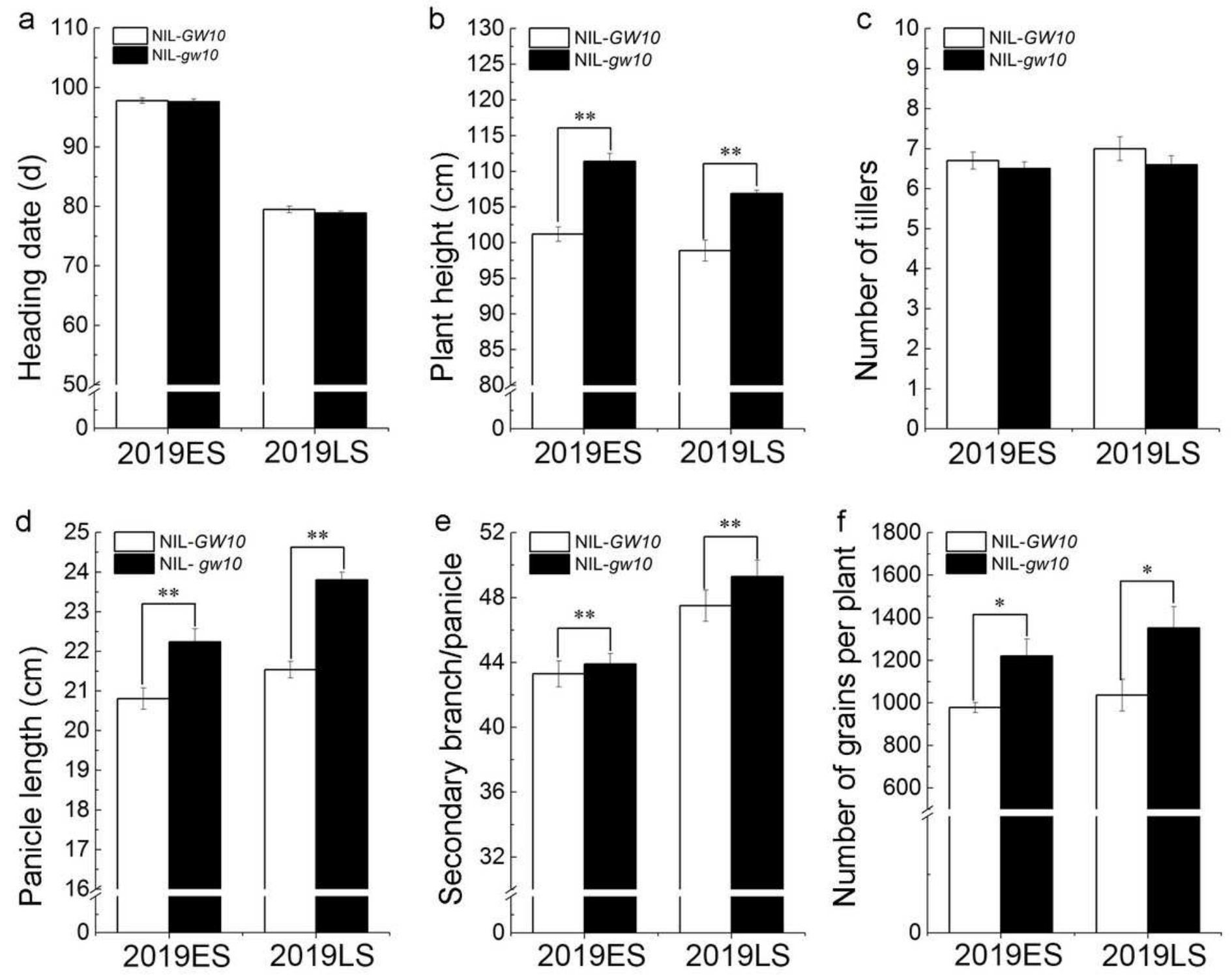

Figure 2

The agronomic traits of NIL-GW10 and NIL-gw10. a. Heading date. b. Plant height. c. Number of effective tiller. d. Panicle length. e. Secondary branches per panicle. f. Number of grains per plant. 2019ES, the early season in 2019; 2019LS, the late season in 2019. Values are means $\pm S D$. ${ }^{*} P \leq 0.05 * * P \leq 0.01$. 


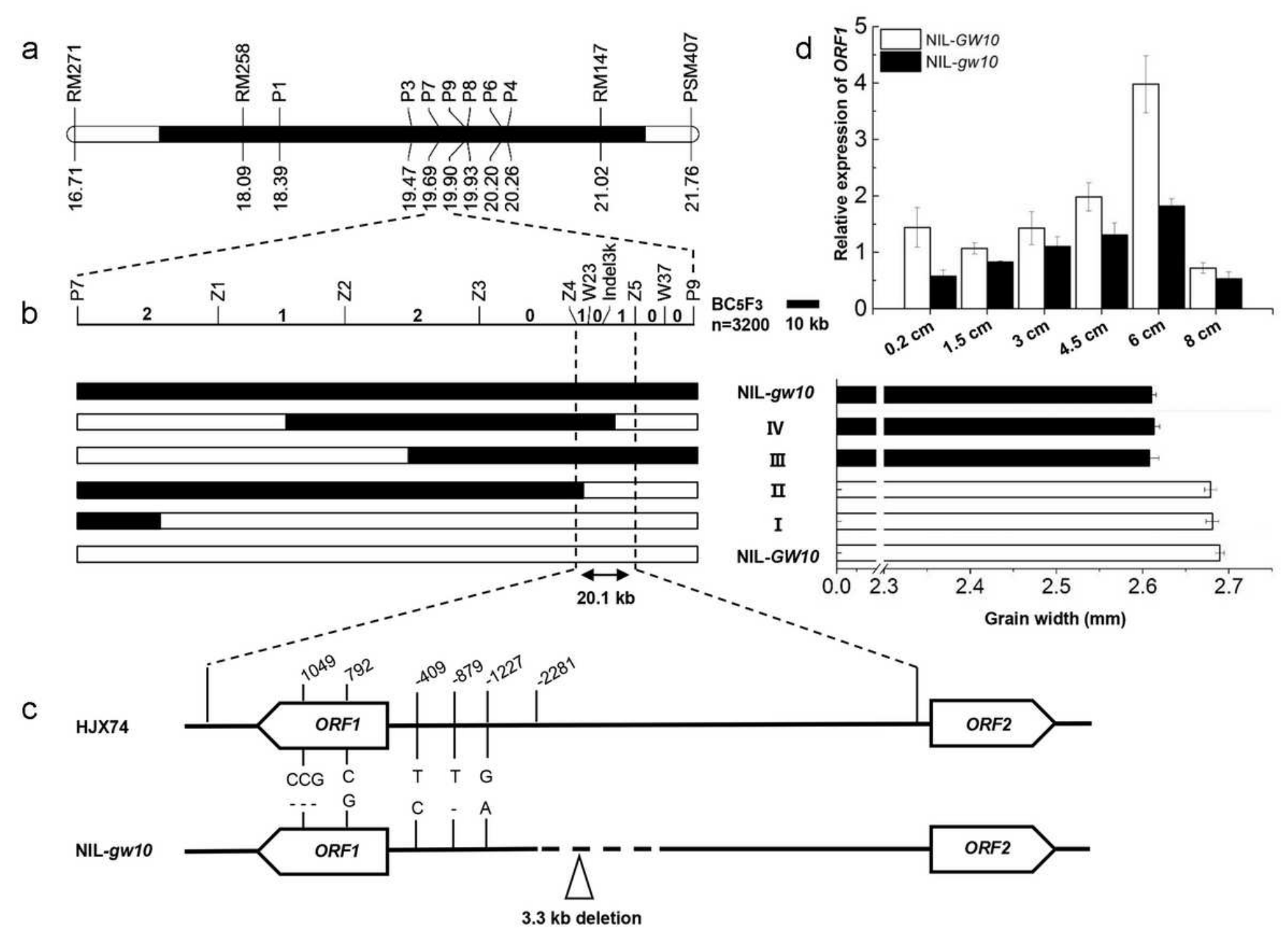

\section{Figure 3}

Fine-mapping of GW10. a. The substitution segment on chromosome 10. b. qGW10 was mapped to 20.1 $\mathrm{kb}$ region between marker $\mathrm{Z} 4$ and $\mathrm{Z} 5$ using 3,200 BC5F3 plants. The number above the line indicate the number of recombinants between each of the two adjacent markers. Black filled and open bars represent substitution segments homozygous for the Lemont and HJX74 alleles, respectively. Grain width is shown for recombinant plants (IIV) and NILs. Data are shown as means $\pm S D(n \geq 100)$. c. Allelic variation in the region of candicate gene ORF1 (Os10g0515400) between Lemont and HJX74. d. Expression of ORF1 in

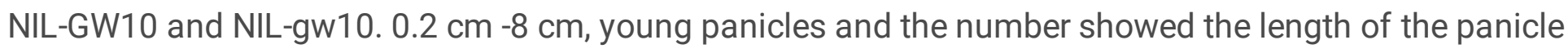
$(\mathrm{cm})$. 

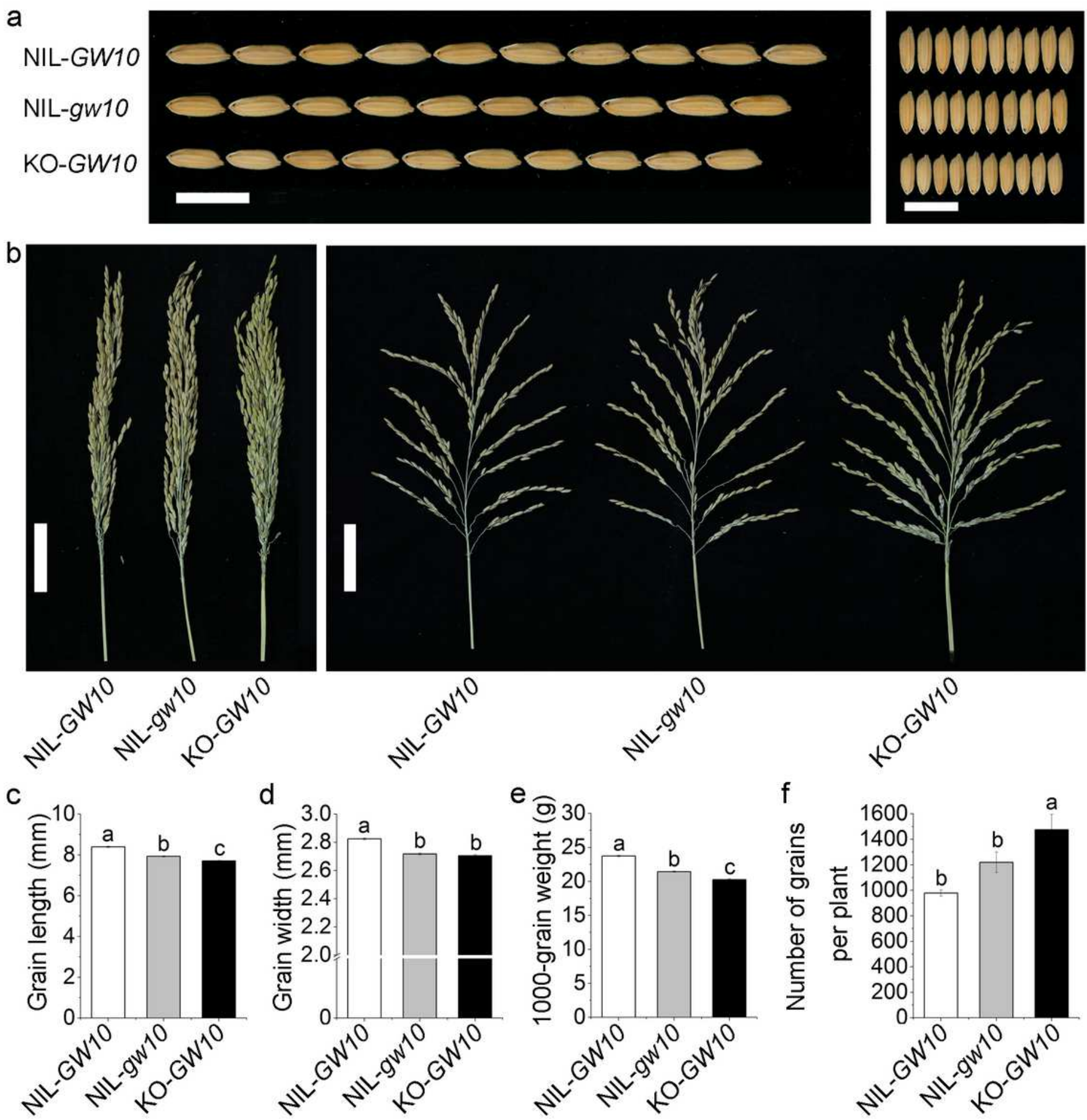

Figure 4

The effects of GW10 on grain size and grain number. a. The grains from NIL-GW10, NIL-gw10 and targetgene edited NIL-GW10 (KO-GW10). Scale bar, $5 \mathrm{~mm}$. b The grains from NIL-GW10, NIL-gw10 and KOGW10. Scale bar, $5 \mathrm{~cm}$. c. Grain length $(n \geq 100)$. d. Grain width $(n \geq 100)$. e. 1000-grain weight, 3 repeats. $f$. number of per plants $(n \geq 20)$. Values shown in $c-f$ are means \pm SD. $a, b, c P \leq 0.05$. 

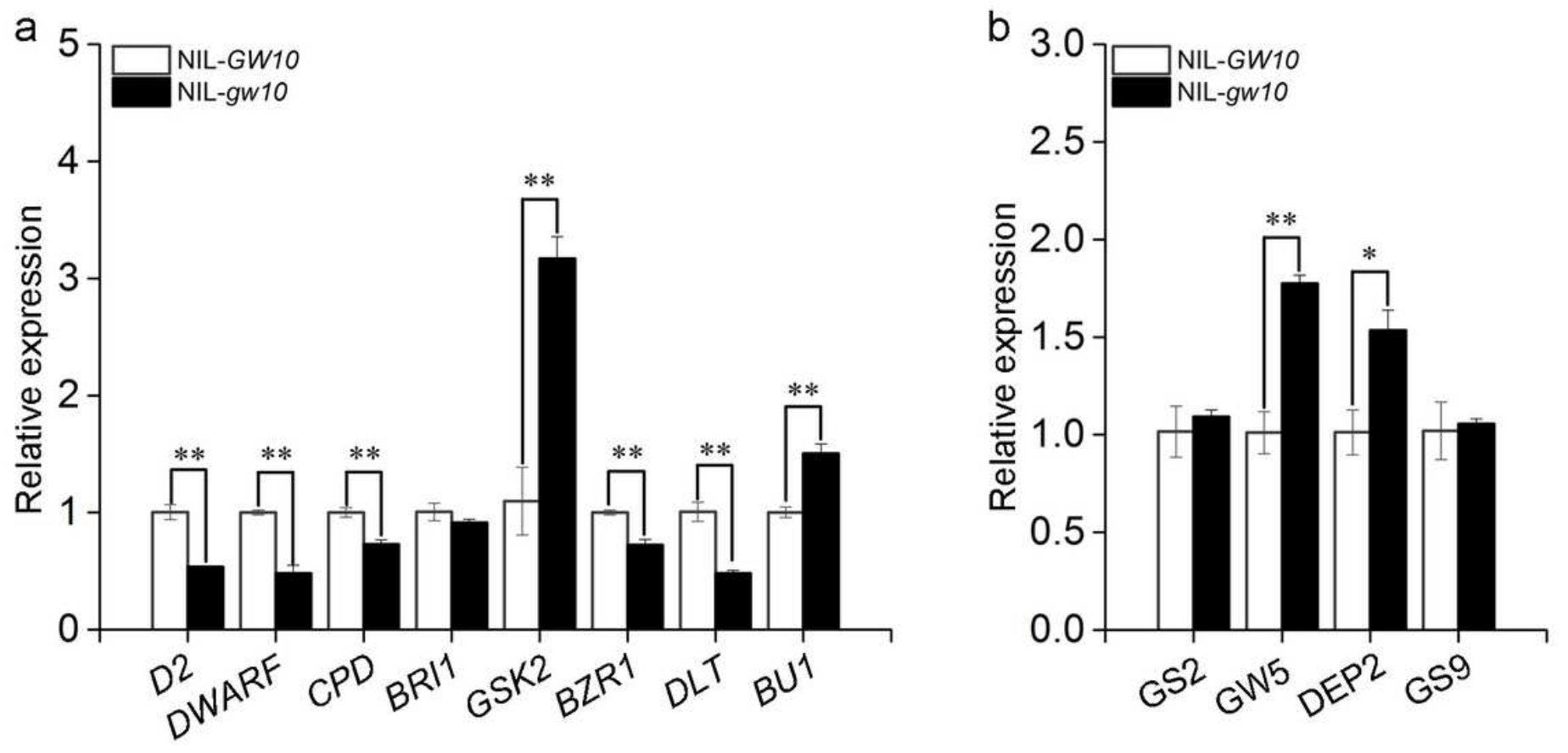

Figure 5

Transcript levels of BR responsive and BR-related grain size genes in young panicle. a BR responsive genes. b. BR-related grain size genes. Expression is shown relative to that of NIL-GW10 plants, which was set to 1. Data are shown as means $\pm S E$. $P$ values from the $t$-test were indicated, ${ }^{*} P \leq 0.05, \star \star P \leq 0.01$

\section{Supplementary Files}

This is a list of supplementary files associated with this preprint. Click to download.

- Supplementarydata1.pdf 